\title{
Atomic-scale Mechanisms of Helium Bubble Hardening in Iron
}

\author{
Yuri N. Osetsky* and Roger E. Stoller \\ Materials Science and Technology Division, Oak Ridge National Laboratory \\ Oak Ridge, TN 37831 USA
}

Copyright notice: This manuscript has been authored by UT-Battelle, LLC under Contract No. DE-AC0500OR22725 with the U.S. Department of Energy. The United States Government retains and the publisher, by accepting the article for publication, acknowledges that the United States Government retains anon-exclusive, paidup, irrevocable, world-wide license to publish or reproduce the published form of this manuscript, or allow others to do so, for United States Government purposes. The Department of Energy will provide public access to these results of federally sponsored research in accordance with the DOE Public Access Plan (http://energy.gov/downloads/doepublic-access-plan). 


\begin{abstract}
Generation of helium due to $(n, \alpha)$ transmutation reactions changes the response of structural materials to neutron irradiation. The whole process of radiation damage evolution is affected by He accumulation and leads to significant changes in the material's properties. A population of nanometric He-filled bubbles affects mechanical properties and the impact can be quite significant because of their high density. Understanding how these basic mechanisms affect mechanical properties is necessary for predicting radiation effects. In this paper we present an extensive study of the interactions between a moving edge dislocation and bubbles using atomicscale modeling. We focus on the effect of He bubble size and He concentration inside bubbles. We found that ability of bubbles to act as an obstacle to dislocation motion is close to that of voids when the He-to-vacancy ratio is in the range from 0 to 1 . A few simulations made at higher He contents demonstrated that the interaction mechanism is changed for over-pressurized bubbles and they become weaker obstacles. The results are discussed in light of post-irradiation materials testing.
\end{abstract}

* Corresponding author.

e-mail: osetskiyyn@ornl.gov 


\section{Introduction}

Generation of helium in materials under neutron irradiation occurs due to $(n, \alpha)$ transmutation reactions [1]. This phenomenon significantly changes the whole microstructure evolution for the commercially interesting temperatures above $600 \mathrm{~K}$ and is a particular concern for future fusion applications due to elevated temperatures and high He-generation rates [2-3]. The need to develop predictive models for He effects has led to extended research programs worldwide (see e.g. [4].) According to the issues considered, studies of He effects can be generally considered in two categories.

One aspect is the prediction of He effects on microstructure evolution. This includes understanding the state of the He in the material and in bubbles, the interactions between mobile defects (vacancies, interstitial atoms) with He atoms and He-filled bubbles, and their impact within the appropriate models for microstructure evolution. There have been numerous studies of how helium generation influences cavity and other microstructural evolution using a range of simulation methods [5-8]. Recently we employed molecular dynamics simulations to study the behavior of $\mathrm{He}$ inside bubbles in an Fe matrix and its dependence on bubble size and temperature; this work led to the development of a modified equation of state for helium in bubbles [9]. In particular, it was found that the equilibrium He-to-vacancy (He/Vac) ratio obtained for bubbles 2 to $5 \mathrm{~nm}$ in diameter is about $0.4-0.7$ at room temperature and weakly decreases with increasing temperature. This is quite consistent with recent experiments where the $\mathrm{He} / \mathrm{Vac}$ ratio for small bubbles in ferritic-martensitic steels was determined using electron energy loss spectroscopy [10,11]. For example, helium density results for a $1.3 \mathrm{~nm}$ bubble lead to a He/vacancy ratio of 0.6 [10]. For bubbles from 2 to $5 \mathrm{~nm}$, the He/vacancy ratio was in the range of $0.25-0.85$ [11]. It is clear that He/Vac ratio depends on the irradiation temperature, rates of atomic displacements and He generation. However these numbers are an estimation of the $\mathrm{He} / \mathrm{Vac}$ ratios to be considered.

The second aspect is the study of $\mathrm{He}$ as an impurity and in bubbles on mechanical properties. Although the first related modeling studies were reported quite a long time ago [12], this issue is much less explored for it demands quite a large-scale computing effort to receive robust results. The series of publications by Hafez Haghighat and Schaublin [13-16] is the most 
comprehensive research to date related to He bubble hardening in iron. The following conclusions can be formulated on the basis of results presented in [13-16]: 1) He-filled bubbles are strong obstacles consistent with corresponding size voids at $\mathrm{He} / \mathrm{Vac} \leq 2$ and stronger than voids at $\mathrm{He} / \mathrm{Vac}>2$; 2) bubble strength decreases at higher temperatures; and 3) there are certain quantitative but not qualitative effects on the bubble obstacle strength at different $\mathrm{He} / \mathrm{Vac}$ and temperatures when different Fe-matrix potentials [17-19] were used together with either the Wilson-Johnson [20] or Juslin-Nordlund [21] pair potentials for He-Fe interactions.

There are several aspects of this previous modeling work that should be mentioned. First, relatively small model crystals were used; as a result, the maximum dislocation length was 17 $\mathrm{nm}$ and the distance between dislocations in the periodic array was $25 \mathrm{~nm}$ even for large $7 \mathrm{~nm}$ diameter obstacles. Second, the quite high strain rate applied (the slowest reported is equal to $3 \times 10^{8} \mathrm{~s}^{-1}$ ) resulted in a dislocation velocity equal to $60 \mathrm{~m} / \mathrm{s}$. Third, very high $\mathrm{He} / \mathrm{Vac}$ ratios of 1 to 5 were modeled. Hafez Haghighat and co-workers also studied the state of bubbles with $\mathrm{He} / \mathrm{Vac}>1$ and reported that a $2 \mathrm{~nm}$ bubble creates Frenkel pair for $\mathrm{He} / \mathrm{Vac}>3$ [22]. This indicates that these bubbles are mechanically unstable.

Based on these observations, we conclude that many important aspects of dislocation interactions with helium bubbles require further attention to clarify the mechanisms involved. Among the most obvious issues are the following:

- Determining the obstacle strength of bubbles in the experimentally reasonable range of He content, i.e. $\mathrm{He} / \mathrm{Vac}$ ratios $<1$,

- Understanding the sensitivity of the modeling results to the applied modeling parameters such as crystal size, strain rate and temperature,

- The atomic scale details of dislocation-bubble interaction mechanisms, and

- The stability and structure of over-pressurized bubbles with high He/Vac ratios, their temperature dependence and effects on bubble - dislocation interactions.

Some of these issues were studied in the present research. However, we focus on one temperature, $\mathrm{T}=300 \mathrm{~K}$, because the majority of experimental mechanical testing has been performed at room temperature. Unlike the work reported in [13-16, 22], we modeled bubbles with a relatively low He content, mainly $\mathrm{He} / \mathrm{Vac} \leq 1$. According to the work reported in Ref. [9], such ratios are consistent with equilibrium bubbles under relevant experimental conditions. We report only a few cases with $\mathrm{He} / \mathrm{Vac}=2$ to demonstrate the dramatic change in the interaction 
mechanism due to over-pressurized conditions. Special attention was paid to the choice of modeling conditions and estimating the accuracy of the modeling results.

\section{Atomic scale model}

The atomic scale model we have used to study the $1 / 2<111>$ (110) edge dislocation interaction with He-filled bubbles is based on the molecular dynamics (MD) model described in [23]. This model describes a periodic array of dislocations and obstacles. The dislocation length, or equivalently, the distance between obstacles along the dislocation <112> line, is one of the most important modelling parameters. It should be long enough to permit the dislocation to adopt the necessary curvature at the critical state, i.e. before it is released from the obstacle. When interacting with strong obstacles, an edge dislocation usually adopts an "Orowan-like" configuration with an extended screw dislocation dipole formed in the obstacle's vicinity. This configuration may be called "Orowan-like" because it is similar to the dipole configuration that occurs for the true Orowan mechanism which involves impenetrable obstacles. It is important here that the interactions of screw segments belonging to the dipole are much stronger the interactions through the periodic boundaries along the dislocation line. In other words, the simulated dislocation segment length, $L$, should be much longer than the largest simulated obstacle diameter, $D_{O}$. In the present research we have used the constant $L=42 \mathrm{~nm}$ for the obstacle size up to $D_{O}=6 \mathrm{~nm}$. Another important parameter is the crystal size along the $\boldsymbol{b}=$ $1 / 2<111>$ Burgers vector, $L_{\boldsymbol{b}}$. The importance of this parameter also arises because of the "Orowan-like" interaction mechanism; the length of the screw dislocation dipole at the critical state should be much smaller than $L_{b}$ to minimize the contribution from the through-boundary interaction along the Burgers vector. In the present research the $L_{b}$ value was chosen depending on the obstacle size and varied from $\sim 30 \mathrm{~nm}$ to $\sim 90 \mathrm{~nm}$ for obstacles from $1 \mathrm{~nm}$ to $6 \mathrm{~nm}$ in diameter. The crystal height, the measurement in the $\langle 110\rangle$ normal direction to the dislocation slip plane, was chosen to be constant, $H=20 \mathrm{~nm}$, for all the obstacles according to estimations described in [22]. The total number of lattice sites in the mobile crystals simulated was therefore varied from 2,074,024 to 6,236,064 depending on the obstacle size. The constant value of $L$ allows direct comparison of the strength of all the obstacles. To generate force to move the dislocation, we have applied the strain rate loading described in [23]. We have used the constant 
strain rate value, $\dot{\varepsilon}=5 \times 10^{6} \mathrm{~s}^{-1}$. The dislocation velocity depends on the applied strain rate and dislocation density and in the applied model can be estimated as: $v_{D}=\dot{\varepsilon} L_{\boldsymbol{b}} H / \boldsymbol{b}$ and varied from $\sim 16 \mathrm{~m} / \mathrm{s}$ to $\sim 49 \mathrm{~m} / \mathrm{s}$ in the smallest and largest crystals, respectively. The applied strain generates a shear deformation of the modeled crystal which in turn generates shear stress (see [22] for how to calculate the shear stress value) which forces a dislocation to move. In MD modeling the instantaneous stress fluctuates depending on the temperature and dislocation velocity (i.e. applied strain rate.) Fig. 1 shows the variation of the instantaneous shear strain, $\tau$, calculated every 100 time steps (the time step for motion equation integration is equal to $2 \mathrm{fs}$ ) during the steady state motion of a $1 / 2<111>\{110\}$ edge dislocation under $5 \times 10^{6} \mathrm{~s}^{-1}$ applied strain rate modeled at $300 \mathrm{~K}$ over $2.5 \mathrm{~ns}$. One can see that its value fluctuates from <-20 MPa to almost 40 MPa. Statistical treatment resulted in the mean value $\bar{\tau}=3.53 \mathrm{MPa}$ (solid line in Fig. 1) and a standard deviation $\sigma=9.8 \mathrm{MPa}$ (the values of $(\bar{\tau} \pm \sigma)$ are indicated by point-dash lines in Fig. 1). Here we accepted the standard deviation as providing the most accurate estimate of the uncertainty in the mean shear stress. However, in the case of dislocation-obstacle interactions the uncertainty in the shear stress estimate should be higher because this process is far from the case of equilibrium steady state dislocation motion considered in Fig. 1. It is difficult to estimate this uncertainty for it depends on the temperature and applied strain rate. We cannot estimate the mean shear stress by a simple time average as in Fig. 1 for the interaction process is limited in time. More important is the obstacle release stress that exists over a limited and rather short time depending on the strain rate applied. As an example we illustrate the typical stress-strain, $\tau(\varepsilon)$, dependence obtained when modeling the interaction between a moving dislocation and a $2 \mathrm{~nm}$ bubble with $\mathrm{He} / \mathrm{Vac}=0.5$. The grey line presents the instantaneous shear stress calculated every 100 time steps. One can see that stress fluctuations are rather big and quite similar to that in Fig. 1 for the steady-state dislocation motion. To extract a reasonably averaged instantaneous value of $\tau$ we used adjacent average (AA) processing of the instantaneous data. The AA processing data with 5, 10 and 20 neighboring points (AA5, AA10 and AA20) are shown by green, black and red lines in Fig. 2. Data for AA20 show reasonably smooth behavior. We used this treatment to produce all of the results reported here, assuming as well that the uncertainty in estimating the shear stress values is the same as for the steady state dislocation motion at the same temperature where its lower limit is $>10 \mathrm{MPa}$. Note that processing parameters (the number of neighboring points) and the shear stress uncertainty (standard deviation of the mean stress) depend upon the 
model strain rate and temperature; both values decrease at low temperature and $\dot{\varepsilon}$, and increase at higher temperatures and $\dot{\varepsilon}$. The maximum on the processed $\tau(\varepsilon)$ curve indicates the critical resolved shear stress (CRSS) which is the measure of the obstacle strength for a given interaction geometry. As shown in [23], if the dislocation segment, $L$, is long enough then CRSS $\propto 1 / L$ for a given obstacle.

The atomic-scale interaction mechanisms were assessed using an on-the-fly visualization during simulation. For the dislocation line visualization we have used two techniques, either analyzing the common neighbors or the atomic disregistry in the dislocation core. The details can be found in [23].

To complete the description of the model accuracy, we consider one more issue related to the equilibration and annealing of simulated gas-filled bubbles: Any defect created in atomicscale modeling requires a certain period of annealing to reach the equilibrium state. The Hefilled bubble is an example that needs a long time to reach equilibrium depending on the size and $\mathrm{He} / \mathrm{Vac}$ ratio as was reported recently in [9]. The need for a long equilibration time is demonstrated in [9], 1-2 ns, to accurately estimate the value of He pressure inside small bubbles with low $\mathrm{He} / \mathrm{Vac}$ ratios. For larger bubbles with higher $\mathrm{He} / \mathrm{Vac}$ ratios, equilibration can be reached faster. The dynamics of temperature and pressure equilibration for He atoms inside a 4 $\mathrm{nm}$ bubble with $\mathrm{He} / \mathrm{Vac}=0.4$ is shown in Fig. 3. In this case the bubble reaches its equilibrium state sometime between 50 and 100 ps. In this study we annealed each crystal with the dislocation and bubble over this time before strain was applied.

For all simulations in this research we have used the following set of interatomic potentials: the embedded atom model potential for Fe from [17], the three-body potential for FeHe interaction from [24, 25] and the pair potential for He-He interaction from [25]. The threebody He-Fe potential was fitted to a wide set of first principles data with the main emphasis on the properties of He-vacancy clusters [26]. This set of potentials was successfully applied in extensive studies of He-vacancy clusters, He-bubble nucleation, growth and stability and He transport in bcc Fe [9, 25-29].

In this paper we report data for 1,2, 4 and $6 \mathrm{~nm}$ diameter bubbles that contain up to 9640 vacancies and the corresponding number of He-atoms when the He/Vac ratio is varied from 0 to 1. The He/Vac ratio was extended up to 2 in the case of $2 \mathrm{~nm}$ bubbles to demonstrate when the interaction mechanism changes. 


\section{Results and discussion}

The results for 1, 2, 4 and $6 \mathrm{~nm}$ bubbles are summarized in Fig. 4 which show the dependence of the CRSS on the He/Vac ratio. The size dependence is strong and follows that observed earlier for voids. Moreover, within the presented values of $\mathrm{He} / \mathrm{Vac}$, the dislocationbubble interaction mechanism is the same and very close to that of voids. To demonstrate this, we present a set of dislocation line configurations at some characteristic stages during its interaction with a $4 \mathrm{~nm}$ bubble with $\mathrm{He} / \mathrm{Vac}=1$ in Fig. 5. These states are fully compatible with similar states for the void described in [13]. In Fig. 5, State 1 is related to dislocation motion through the crystal before it comes into contact with the bubble. This is equivalent to the initial stage of $\tau(\varepsilon)$ curve shown in Fig. 2 when the shear stress is eventually constant. State 2 occurs when the dislocation is attracted by the bubble and comes into contact. This state is characterized by a strong attractive force and is accompanied with the sudden drop of shear stress to the negative value in Fig. 2. When dislocation intersects the bubble it loses a certain amount of dislocation line length. This is energetically favorable for the dislocation line energy inside the bubble can be set to 0 (a similar effect is demonstrated for a void in [13].) At State 3, the dislocation crosses the bubble along its diameter and therefore loses the longest possible segment. This is the minimum energy and a zero stress state. It is equivalent to the point when $\tau(\varepsilon)$ curve intersects zero stress level at about $\varepsilon=0.25$ in Fig. 2. Further straining leads to dislocation glide over the bubble surface, restoring the dislocation line in the crystal and creating a step on the exit surface of the bubble. During this process the shear stress increases and dislocation line curve due to glide of the segments in the crystal. State 4 in Fig. 5 shows the critical shape of the dislocation just before it breaks out. The considered obstacle is "strong" and the initial edge dislocation creates a relatively long dipole of screw segments with a near-zero angle between them. As mentioned above, the dislocation is similar to that obtained by the Orowan mechanism for impenetrable obstacles. The only difference between this and the true Orowan mechanism is the distance between dipole segments. In the case of impenetrable obstacles, this is equal to the obstacle diameter in the dislocation slip plane. In the case of bubbles and voids, this can be much shorter due to the dislocation glide over the bubble surface and the screw segments cross-slip. The State 4 in Fig. 5 is characterized by the maximum value 
on the $\tau(\varepsilon)$ curve. Finally, State 5 occurs when the dislocation is released from the bubble and glides through the crystal.

The mechanism described above was observed for all the bubbles with $\mathrm{He} / \mathrm{Vac} \leq 1$. According to Fig. 5, and considering the uncertainty in the CRSS value definition of $>10 \mathrm{MPa}$, the dependence of CRSS is rather weak for $\mathrm{He} / \mathrm{Vac} \lesssim 0.8$. However, we observed some differences between the interactions with low- and high- He content bubbles. One example of these differences is demonstrated in Fig. 6, which shows the dislocation line shape after it is released from a $4 \mathrm{~nm}$ bubble with different $\mathrm{He} / \mathrm{Vac}$. At $\mathrm{He} / \mathrm{Vac}=0$, i.e. a void, the line eventually climbs up absorbing vacancies from the obstacle. Visualization of this interaction has shown that one of the two screw segments that terminate at the void surface (see i.e. State 4 in Fig. 5) crossslips up creating the corresponding superjog after the dipole is released from the void. A similar mechanism for other voids in Fe was discussed in [30]. However, a monotonic increase in $\mathrm{He} / \mathrm{Vac}$ ratio reduces the number of absorbed vacancies, and at $\mathrm{He} / \mathrm{Vac}=1$, the dislocation line absorbs more atoms and climbs down (see the corresponding dislocation line in Fig. 6). In this case one of the two screw dislocation segments climb down declines rather than up at lower $\mathrm{He} / \mathrm{Vac}$ values.

As was pointed out in [30] such climb reduces the CRSS; however, the mechanism is not yet understood. The data obtained here, e.g. the CRSS (Fig. 4) and dislocation line shapes (Fig. 6) are in principle consistent with the maximum CRSS values usually being correlated with the minimum climb (and therefore the weakest cross-slip). However, this conclusion should be verified at other conditions, preferably at lower temperature and strain rate, because of the high uncertainty in CRSS values. The only confident conclusion we can make is that at the highest $\mathrm{He}$ content studied here, $\mathrm{He} / \mathrm{Vac}=1$, all the bubbles are weaker than the corresponding voids.

To explore the effect of high He concentration, we modeled a $2 \mathrm{~nm}$ bubble with $\mathrm{He} / \mathrm{Vac}=2$. The $\tau(\varepsilon)$ curves for some He/Vac values are presented in Fig. 7 and the corresponding dislocation line shapes in Fig. 8. Obvious qualitative differences are observed in the both figures. In Fig. 7 the $\tau(\varepsilon)$ curve for $\mathrm{He} / \mathrm{Vac}=2$ is completely different from all the others with $\mathrm{He} / \mathrm{Vac} \leq 1$. The corresponding line shape demonstrates a significant amount of climb down that is equivalent to 36 atoms absorbed by the line. This means that the $2 \mathrm{~nm}$ bubble, that occupies 339 vacancy sites, increased its volume by more than $10 \%$. Visualization of the interaction mechanisms has confirmed that it is qualitatively different from all other cases considered so far. During the 
equilibration process the bubble shape changed from the high symmetric sphere to some irregular shape that expanded locally. This occurred because for $\mathrm{He} / \mathrm{Vac}=2$ at $\mathrm{T}=300 \mathrm{~K}$ the bubble is strongly over-pressurized and the bubble surface is deformed significantly outwards toward the Fe matrix but no clear punching of interstitial atoms was observed at this stage. However, when the dislocation approached the bubble it "emitted" an interstitial cluster that attracted the dislocation. This can be seen as a sudden drop on the corresponding $\tau(\varepsilon)$ curve on Fig. 7. The cluster was absorbed by the line creating a superjog and the dislocation was repelled from the bubble. At this state the corresponding $\tau(\varepsilon)$ curve on Fig. 7 abruptly increased. At the next stage, due to continuous straining the jogged dislocation was pushed towards the bubble. However, unlike the other cases when the straight dislocation line intersected the bubble in the equatorial plane, the heavily jogged line just touches the periphery of the deformed bubble. As a result, the dislocation line now does not shear the whole bubble and the CRSS decreases by almost $40 \%$. All these stages can be clearly seen in the video provided in the Supplemental material. It is obvious that the over-pressurized bubble state arising from such a high $\mathrm{He} / \mathrm{Vac}$ ratio is responsible for this change in the interaction mechanism.

We should note that CRSS decrease at $\mathrm{He} / \mathrm{Vac} \simeq 2$ was reported earlier in [13-16]; however, the detailed mechanism and the difference with lower He-content bubbles was not described. Here we confirm the drop in CRSS and identify the mechanism for the case of $2 \mathrm{~nm}$ bubble with $\mathrm{He} / \mathrm{Vac}=2$ at $\mathrm{T}=300 \mathrm{~K}$. We expect a qualitatively similar decrease in CRSS for other sizes, which should be temperature dependent. The higher He-content bubbles studied in [13-16] demonstrated a significant increase in CRSS for up $\mathrm{He} / \mathrm{Vac}=5$, however the interacting mechanisms were not described. We expect that at such a high He-content a bubble should punch out interstitial atoms well before the dislocation approaches it, and in this case the interaction mechanism could be quite different than we observed with $\mathrm{He} / \mathrm{Vac}=2$. The difference is that in the present case the interstitial cluster was released from the bubble due to the strain fields from the approaching dislocation. It was released, a) in the place and b) with the orientation corresponding to the minimum interaction energy. These are, a) the negative pressure volume below the dislocation slip plane, and b) interstitial atom orientation along the dislocation Burgers vector. This correlated formation of an interstitial cluster from the over-pressurized bubble is the mechanism that reduces CRSS for a given size within a certain He/Vac and temperature range. However, if the He/Vac is much higher a strongly over-pressurized bubble may punch single 
interstitials or/and interstitial clusters out during its equilibration independent of the dislocation. In these cases interstitial clusters should be much larger (due to a much higher pressure) and their position and direction do not correlate with the dislocation stress fields. Therefore, dislocationbubble interaction mechanism may be different for it may now involve interaction with the separate large interstitial cluster at the same time. The key issue here is the behavior of strongly over-pressurized bubbles and how the $\mathrm{He} / \mathrm{Vac}$ ratio, temperature and bubble size affect the creation of interstitials atoms, i.e. the number of interstitials, interstitial cluster formation geometry and crystallography. Once these mechanisms are understood their effect on dislocation-bubble interactions can be rationalized using a computational approach similar to that applied here. This work is now in progress.

An important issue in atomistic modeling dislocation-obstacle interactions is the accuracy of the estimated critical stress or obstacle strength. This involves a number of different contributions. One of them, the accuracy of estimating applied stress during dislocation motion, was studied here. It was found that the instantaneous applied stress displays substantial scatter about the mean value and the deviation depends strongly on the temperature with a weaker dependence on the applied strain rate. An standard deviation of the instantaneous applied stress was estimated to be $\pm 10 \mathrm{MPa}$. Another source of an inaccuracy in estimating the obstacle strength is the high strain rate required by molecular dynamics simulations. We employed a strain rate of $5 \times 10^{6} \mathrm{~s}^{-1}$ which provides a dislocation velocity from $\sim 16 \mathrm{~m} / \mathrm{s}$ to $\sim 49 \mathrm{~m} / \mathrm{s}$ in the smallest and largest crystals, respectively. Earlier studies of the effect of dislocation velocity on voids in $\mathrm{Fe}[30]$ has demonstrated that the critical stress saturates at rates below $\sim 10^{6} \mathrm{~s}^{-1}$ while keeping the same interaction mechanism as at a high rate of $10^{8} \mathrm{~s}^{-1}$. A He-filled bubble is a more complicated object than a void so the strain rate sensitivity can be different. Clarification of this issue requires addition simulations at lower strain rates and this work is in progress.

We should also note that the size scale of dislocation-bubble interaction mechanisms is, in principle, compatible with direct transmission electron microscopy in situ straining experiments. It may be possible to study different mechanisms by performing in situ straining at different temperatures and therefore change the bubble pressure. Large-scale atomistic simulations could then reproduce experimental conditions (except for the strain rate) to help in understanding the mechanisms observed. A similar direct modeling experiment comparison was made in previous studies of dislocation interactions with stacking fault tetrahedra when new 
mechanisms were discovered [31].

\section{Summary}

A large-scale atomistic study using molecular dynamics simulations was carried out to investigate the detailed interactions of moving edge dislocations with helium bubbles in iron. The important physical parameters of the computational model and their effects on the accuracy and uncertainty of simulation results were studied. Some of these issues are general, such as the accuracy of the shear stress definition, while others are specific to the behavior of gas-filled bubbles, such as the required equilibration time. The study focused on the interaction between a moving $1 / 2<111>\{110\}$ edge dislocation, and bubbles in the size range of 1 to $6 \mathrm{~nm}$ diameter, with the physically-relevant but so-far unexplored range of He-to-vacancy ratio from 0 to 1 at a temperature of $300 \mathrm{~K}$. The primary results include:

1. These bubbles demonstrate qualitatively the same hardening mechanism as that obtained earlier for voids,

2. The strength of these bubbles was found to be weakly dependent on. He/Vac ratio within the range of 0 to $\sim 0.8$. At higher $\mathrm{He} / \mathrm{Vac}$ ratios the bubble strength decreases.

3. Dislocation climb due to the interaction with bubbles depends on the $\mathrm{He} / \mathrm{Vac}$ ratio. This reflects a smooth change of the interaction mechanisms from that of voids to over-pressurized bubbles that occurs at $\mathrm{He} / \mathrm{Vac} \approx 2$ (for $2 \mathrm{~nm}$ bubble at $\mathrm{T}=300 \mathrm{~K}$ ).

4. The over-pressurized $2 \mathrm{~nm}$ bubble with $\mathrm{He} / \mathrm{Vac}=2$ has demonstrated a completely different strengthening mechanism which is about $40 \%$ weaker than for $\mathrm{He} / \mathrm{Vac} \leq 1$. In this mechanism the over-pressurized bubble emits an interstitial cluster under the influence of the approaching dislocation strain fields. Correlated emission of interstitial clusters minimizes the dislocation-bubble intersection and the maximum shear stress. The mechanism can be clearly seen on the video provided in the Supplement material.

5. The results are compared with those available in the literature and the possible mechanisms for strongly over-pressurized bubbles are discussed. 


\section{Acknowledgements}

Research sponsored by the Office of Fusion Energy Sciences, U.S. Department of Energy, under contract DE-AC05-00OR22725 with UT-Battelle, LLC. 


\section{References.}

1. R. E. Stoller, J. Nucl. Mater. 174 (1990) 289-310.

2. H. Schroeder, P. Batfalsky, J. Nucl. Mater. 117 (1983) 287.

3. T. Yamamoto, G.R. Odette, P. Miao, D.T. Hoelzer, J. Bentley, N. Hashimoto, H. Tanigawa, and R.J. Kurtz, J. Nucl. Mater. 367-370 (2007) 399-410.

4. B.D. Wirth, G.R. Odette, J. Marian, L. Ventelon, J.A. Young-Vandersall, L.A. ZepedaRuiz, J. Nucl. Mater. 103 (2004) 329.

5. N. M. Ghoniem, J. Nucl. Mater. 174 (1990) 168-177.

6. R. E. Stoller and G. R. Odette, "The Effect of Helium on Swelling: Influence of Cavity Density and Morphology," Effects of Radiation on Materials, ASTM STP 782, H. R. Brager and J. S. Perrin, Eds., American Society of Testing and Materials, Philadelphia, 1982, pp. 275-294.

7. J. B. Adams and W. G. Wolfer, J. Nucl. Mater. 166 (1989) 235-242.

8. A. Caro, J. Hetherly, A. Stukowski, M. Caro, E. Martinez, S. Srivilliputhur, L. ZepedaRuiz, M. Nastasi, J. Nucl. Mater. 418 (2011) 261-268.

9. R.E.Stoller and Y.N.Osetsky, J. Nucl. Mat. 455 (2014) 258-262.

10. Y. Wu, G.R. Odette, T. Yamamoto, J. Ciston, P. Hosemann, “An Electron Energy Loss Spectroscopy Study of Helium Bubbles in Nanostructured Ferritic Alloys," Fusion Reactor Materials, Semiannual Progress Report DOE/ER-0313/54, Oak Ridge National Laboratory (2013) 173-179.

11. S. Fréchard, M. Walls, M. Kociak, J.P. Chevalier, J. Henry, D. Gorse, J. Nucl. Mater. 393 (2009) 102.

12. M.I. Baskes, M.S.Daw, "The Effects of Impurities on Dislocation Dynamics and Fracture: An Atomistic Study", $4^{\text {th }}$ International Conference on Hydrogen Effects on Material Behavior (Jackson Lake Lodge, WY: 1989), Published: TMS (September 1989), ISBN-10: 0873391500 .

13. R. Schaublin and Y.L. Chiu, J. Nucl. Mater. 362 (2007) 152. 
14. S.M. Hafez Haghighat and R. Schaeublin, "Molecular dynamics modeling of cavity strengthening in irradiated iron", Proceedings of Third International Conference of Multiscale Materials Modeling, Freiburg, Germany, September 2006, p.729.

15. S.M. Hafez Haghighat and R. Schaeublin, J. Comput. Aided Mater. Des. 14 (2008) 191.

16. S.M. Hafez Haghighat and R. Schaublin, Philos. Mag. 90 (2010) 1075.

17. G.J. Ackland, D.J. Bacon, A.F. Calder and T. Harry, Phil. Mag. A 75 (1997) 713.

18. M.I. Mendelev, S. Han, D.J. Srolovitz, D.Y. Sun and M. Asta, Phil. Mag. 83 (2003) 3977.

19. S.L. Dudarev and P.M. Derlet, J. Phys. Condens. Matter. 17 (2005) 7097.

20. W.D. Wilson and R.A. Johnson, in Rare Gases in Metals, P.C. Gehlen, J.J. Beeler and R.J. Jaffe, eds., Premium Press, NY, 1972, 375.

21. N. Juslin and K. Nordlund, J. Nucl. Mater. 382 (2008) 143.

22. S.M. Hafez Haghighat, G.Lucas and R. Schaublin, European Phys. Lett., 85 (2009) 60008.

23. Yu.N. Osetsky and B.J. Bacon, Model. Simul., Mater. Sci. and Eng. 11 (2003) 427.

24. T. Seletskaia, Yu.N. Osetskiy, R.E. Stoller and G.M. Stocks, J. Nucl. Mater. 361 (2007) 52-61.

25. R.E. Stoller, S.I. Golubov, P.J. Kamenski, T. Seletskaia, Yu.N. Osetsky, Philos. Mag. 90 (2010) 923.

26. R.A. Aziz, A.R. Janzen and M.R. Moldovan, Phys. Rev. Let. 74 (1995) 1586.

27. T. Seletskaia, Yu.N. Osetsky, R.E. Stoller and G.M. Stocks, J. Nucl. Mater. 351 (2006) 109-118.

28. D.M. Stewart, Yu. N. Osetskiy, R.E. Stoller, J. Nucl. Mater. 417 (2011) 1110.

29. D.M. Stewart, Yu.N. Osetsky, R.E. Stoller, S. I. Golubov, T. Seletskaia, P.J. Kamenski, Philos. Mag. 90 (2010) 923.

30. Yu.N. Osetsky and D.J. Bacon, Philos. Mag. 90 (2010) 945.

31. Yu.N. Osetsky, Y. Matsukawa, R.E. Stoller and S.J. Zinkle, Philos. Mag. Lett. 86 (2006) 511. 


\section{Figure captions}

Fig. 1 Modeling of steady state motion of $1 / 2<111>\{110\}$ dislocation at a strain rate of $5 \times 10^{6} \mathrm{~s}^{-1}$ at $300 \mathrm{~K}$ : grey line - instantaneous shear stress calculated every 100 time steps ; solid black line - mean value, $3.8 \mathrm{MPa}$, estimated over $2.5 \mathrm{~ns}$, point-dash lines - mean stress plus/minus standard deviation value, $\sigma=9.8 \mathrm{MPa}$

Fig. 2 Stress-strain curve observed during dislocation interaction with $2 \mathrm{~nm}$ bubble with $\mathrm{He} / \mathrm{Vac}=0.5$ : grey line - instantaneous shear stress calculated every 100 time steps; green, red and black lines - processes by adjacent average over 5, 10 and 20 neighbor points respectively

Fig. 3 Evolution of He-atoms temperature and pressure inside the $4 \mathrm{~nm}$ bubble with $\mathrm{He} / \mathrm{Vac}=1$ during bubble equilibration

Fig. 4 Dependence of the critical resolved shear stress versus He/Vac ratio for bubbles of 1 , 2, 4 and $6 \mathrm{~nm}$ in diameter

Fig. 5 Shape of the dislocation line in the $\{110\}$ slip at different stages during interaction with $4 \mathrm{~nm}$ bubble with $\mathrm{He} / \mathrm{Vac}=1$. Stages 1 to 5 are explained in the text

Fig. 6 Shape of the dislocation line viewed along the Burgers vector after interaction with 4 $\mathrm{nm}$ bubbles with different $\mathrm{He} / \mathrm{Vac}$ ratios

Fig. 7 Stress-strain curves obtained during dislocation interaction with $2 \mathrm{~nm}$ bubbles with different $\mathrm{He} / \mathrm{Vac}$ ratios

Fig. 8 Shape of the dislocation line viewed along the Burgers vector after interaction with 2 $\mathrm{nm}$ bubbles with different $\mathrm{He} / \mathrm{Vac}$ ratios 


\section{Figure}

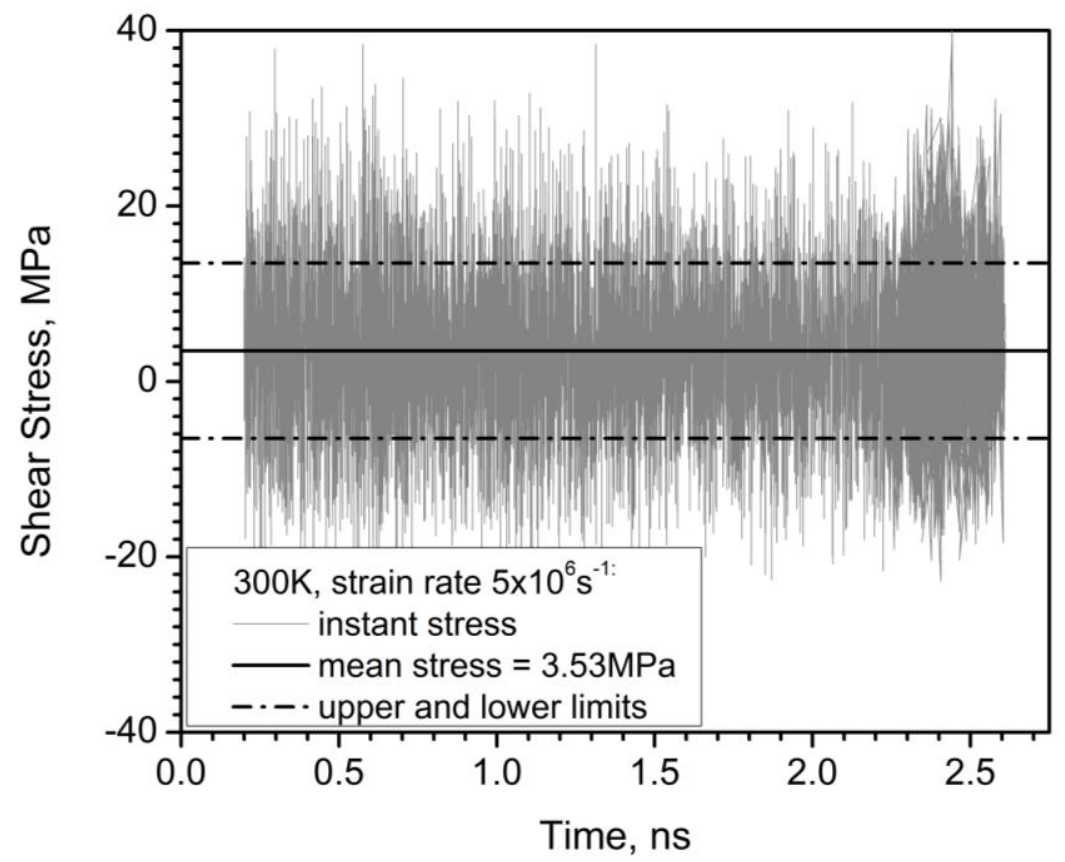

Figure 1

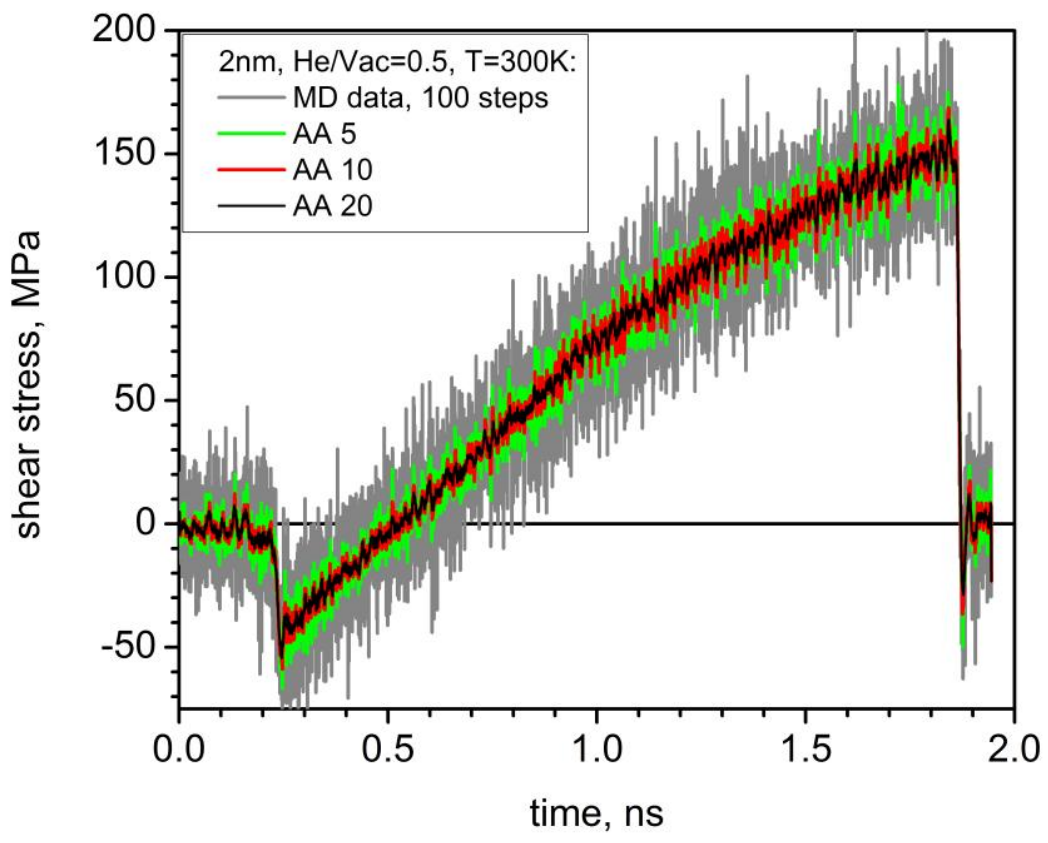

Figure 2 


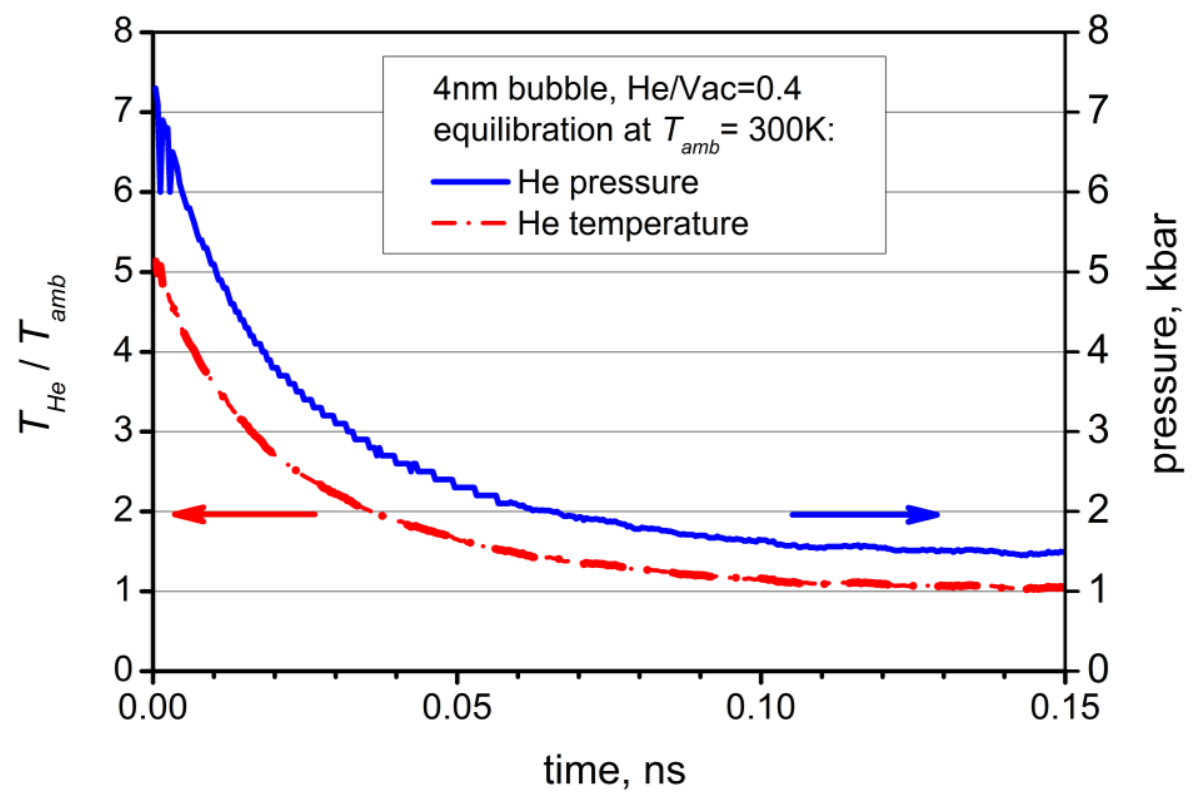

Figure 3

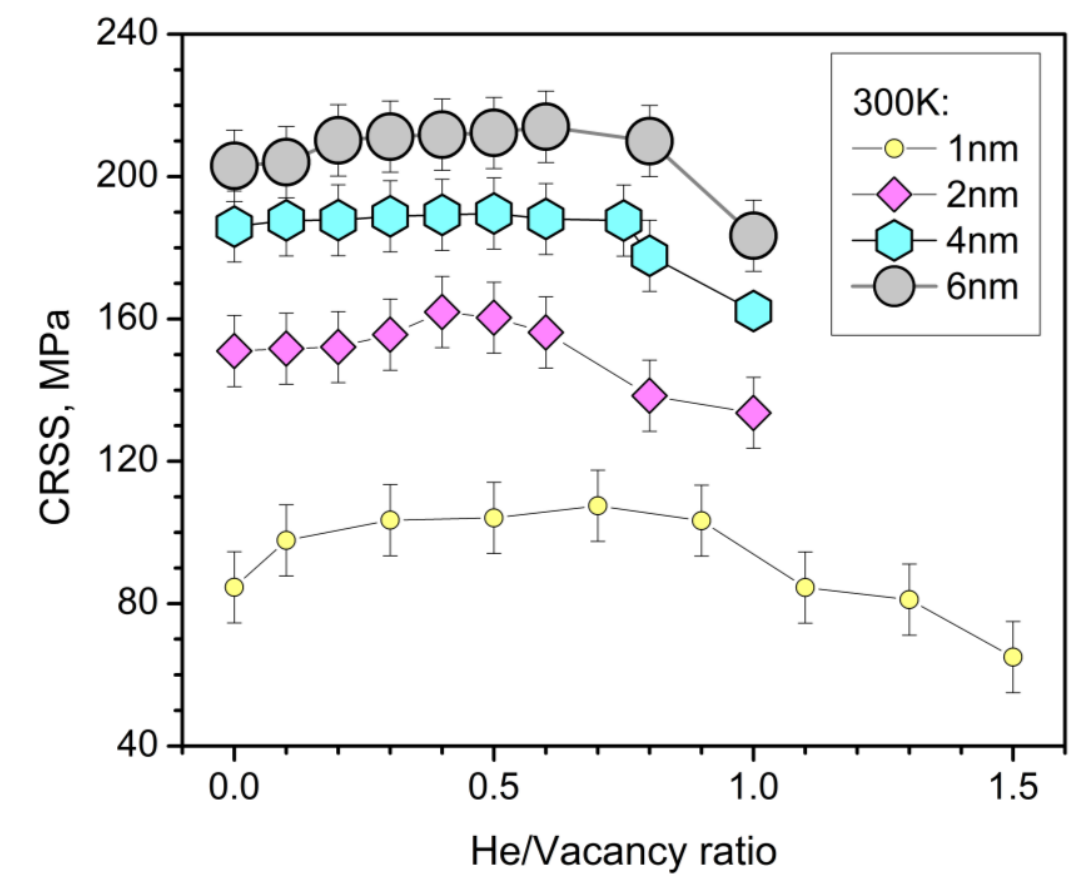

Figure 4 


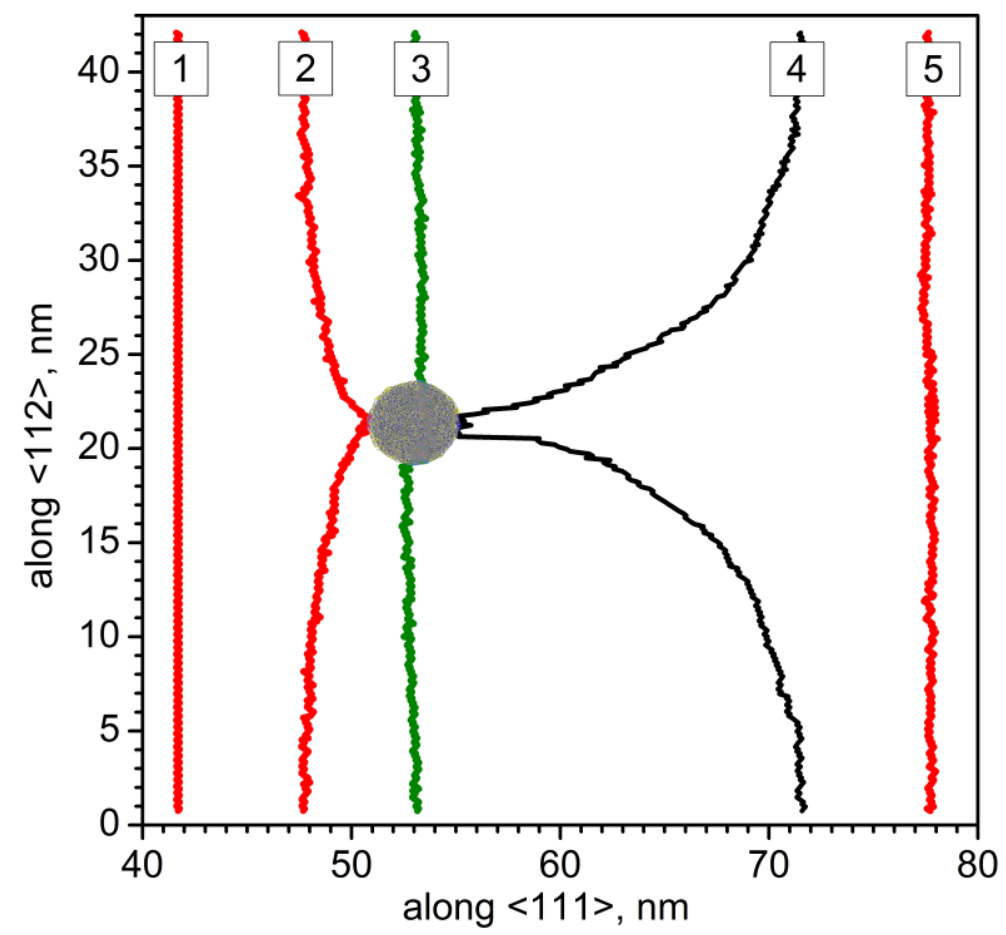

Figure 5

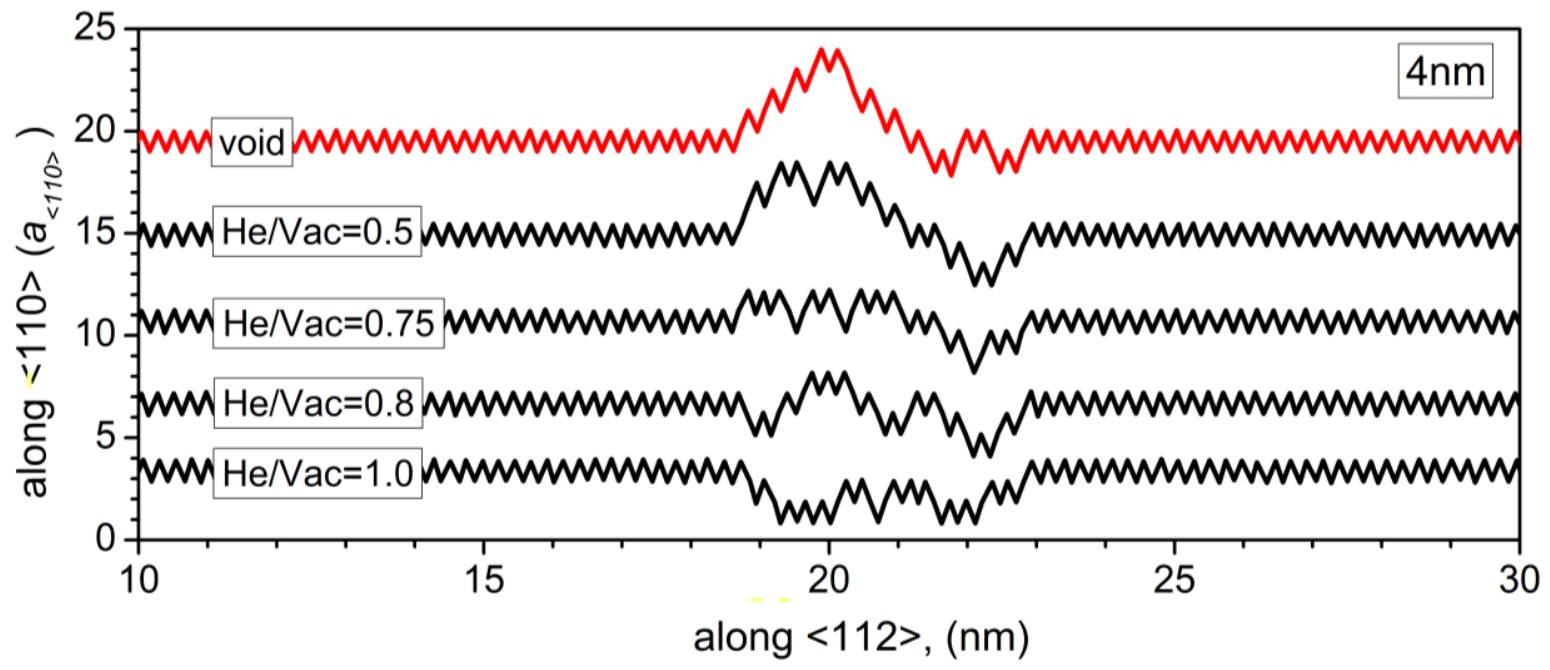

Figure 6 


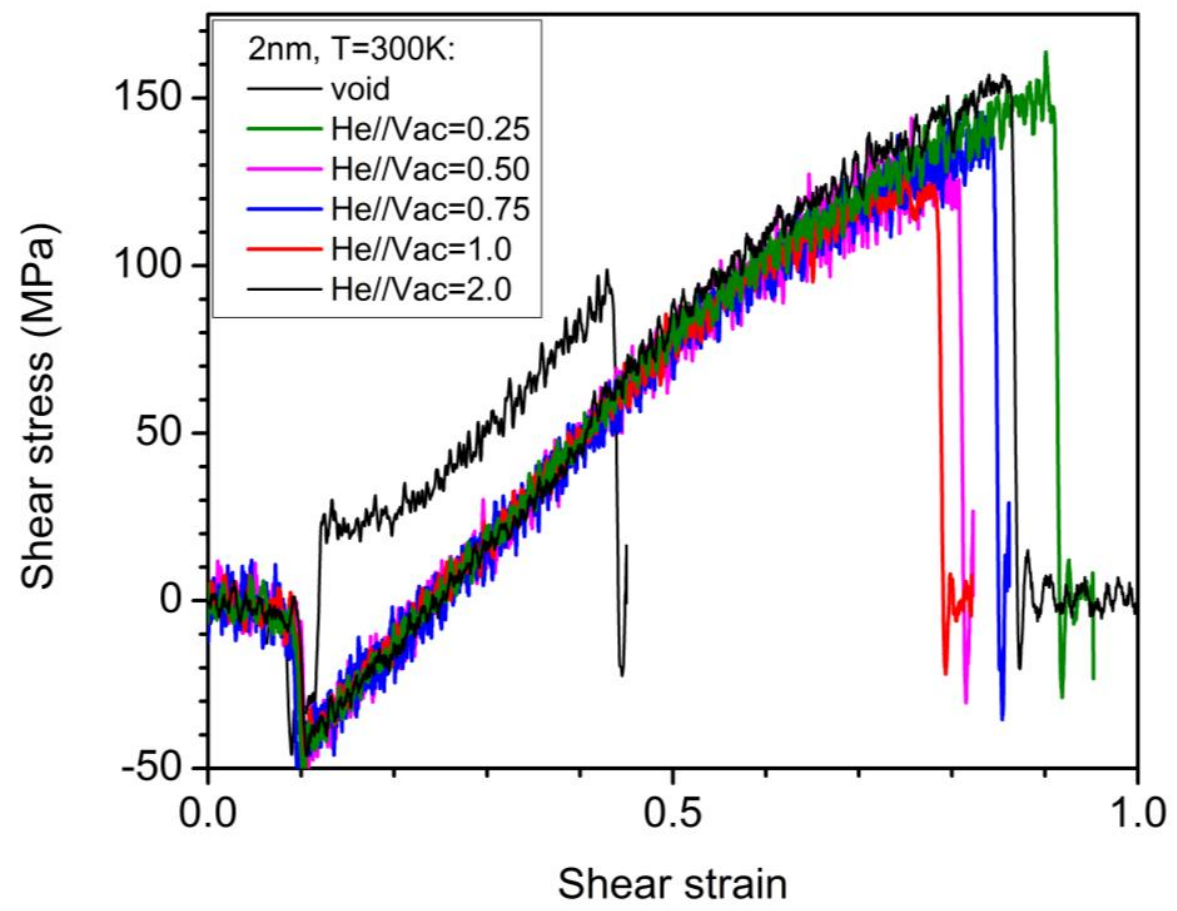

Figure 7

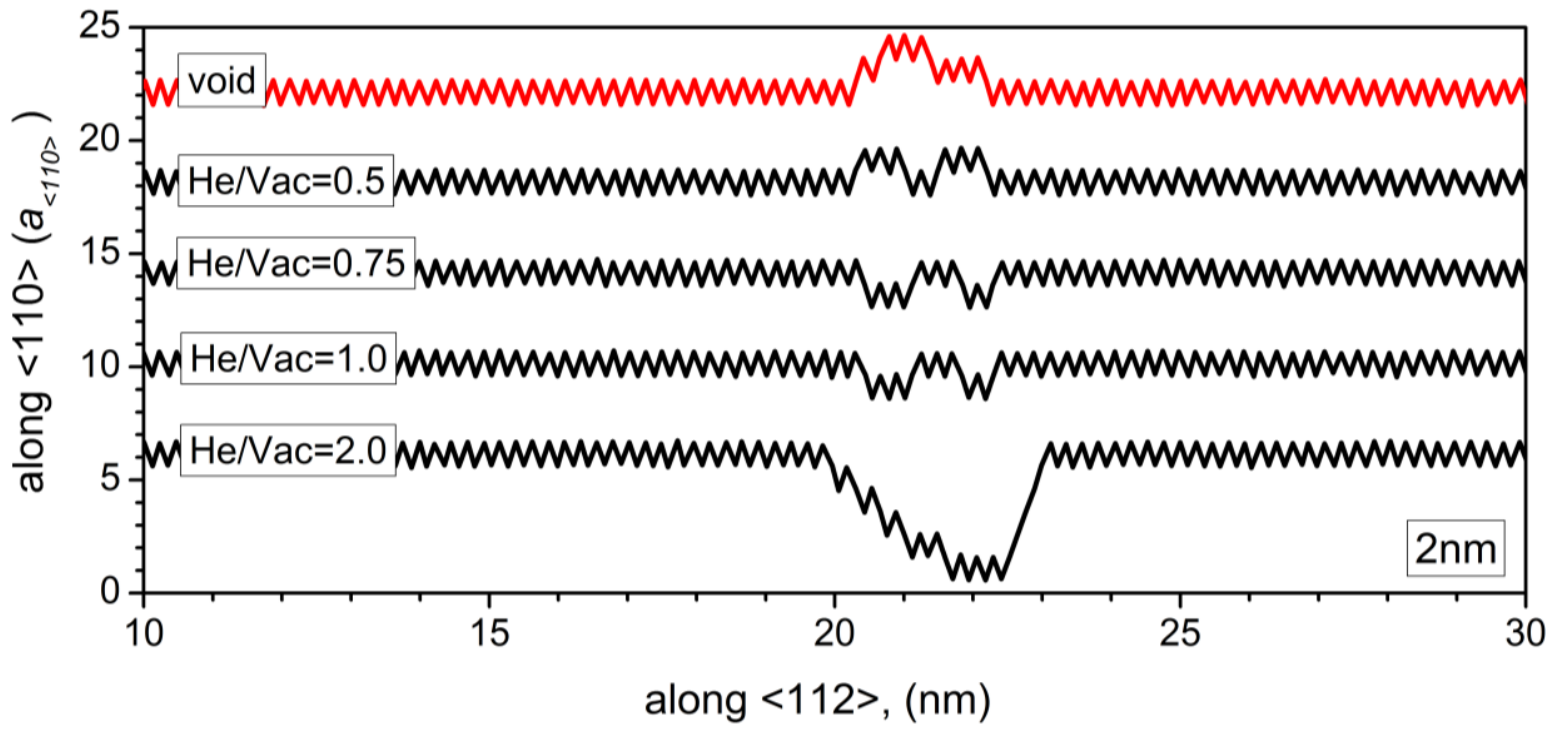

Figure 8 